\title{
Association of Gestational Diabetes Mellitus and Low-density Lipoprotein (LDL) Particle Size
}

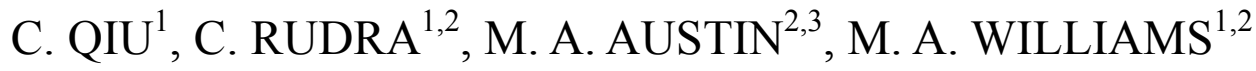 \\ ${ }^{1}$ Center for Perinatal Studies, Swedish Medical Center, ${ }^{2}$ Department of Epidemiology, University of \\ Washington School of Public Health and Community Medicine, ${ }^{3}$ Institute for Public Health \\ Genetics, University of Washington, Seattle, USA
}

Received July 25, 2006

Accepted November 3, 2006

On-line available January 2, 2007

\begin{abstract}
Summary
A predominance of small, dense low-density lipoproteins (LDL) is characteristic of the dyslipidemic state seen in type 2 diabetes. However, no study has investigated the association in gestational diabetes mellitus (GDM), which is pathophysiologically similar to type 2 diabetes. We hypothesized that LDL particle size is reduced in GDM cases compared with controls. Gradient gel electrophoresis was used to characterize LDL subclass phenotypes in non-fasting intrapartum plasma from 105 GDM cases and 96 controls. All participants were free of pre-existing diabetes or hypertension. The authors used logistic regression to estimate odds ratios (OR) and $95 \%$ confidence intervals (CI) adjusted for confounders. Women with this phenotype had a significant 4.9-fold (95\% CI: 1.1-23.2) increased risk of GDM compared with those with the large, buoyant phenotype. The magnitude of this association was attenuated when plasma triglyceride and other confounders were included in the model (OR=4.2, $95 \%$ CI: 0.5-39.5). Mean LDL particle size in GDM cases was smaller compared with controls $(270.1$ vs. $272.7 \AA$, $\mathrm{p}=0.003)$. The OR of GDM risk was 1.8 (95 \% CI: 0.9-3.3) for every 10- $\AA$ reduction in LDL particle size. Large prospective studies are needed to evaluate the association between smaller LDL particle size in early pregnancy with subsequent GDM risk.
\end{abstract}

Key words

Gestational diabetes mellitus • LDL particle size

\section{Introduction}

Gestational diabetes mellitus (GDM), defined as carbohydrate intolerance first recognized during pregnancy, continues to be a common medical complication of pregnancy in the United States and worldwide. The disorder is associated with a high frequency of maternal and fetal morbidity (American
Diabetes Association 1999). Women with a history of GDM are more likely to develop type 2 diabetes and cardiovascular diseases after pregnancy as compared with their counterparts who remained normoglycemic during pregnancy (O'Sullivan 1984). For instance, the cumulative incidence of type 2 diabetes later in life has been reported to range from 22 to $60 \%$ (O'Sullivan 1984, Coustan et al. 1993, Damm 1998) among women with a 
prior history of GDM.

The etiology of GDM has been shown to involve an exaggerated insulin resistance that entails several steps of intracellular insulin signal generation and propagation (Ben-Haroush et al. 2004). Although the mechanisms underlying these processes have not been fully described, both clinical and epidemiological studies illustrate an aggregation of risk factors suggesting a multifactorial process involving both environmental and genetic factors. Notably, some investigators attributed the increased risk of developing type 2 diabetes mellitus and hypertension in women with history of GDM to their dyslipidemic profiles (Enquobahrie et al. 2005, Lauenborg et al. 2005, AbouGhalia et al. 2003).

Low-density lipoprotein (LDL) particles are the major carriers of plasma cholesterol in humans. LDL exhibits substantial heterogeneity based on measures of size, density, and chemical composition. Krauss and Burke (1982), with the use of analytical procedures such as gradient gel electrophoresis and density gradient ultracentrifugation, identified many distinct subclasses of LDL in plasma samples from healthy humans. Austin et al. (1988) subsequently categorized LDL particles into two major distinct phenotypes denoted as A and B. LDL subclass phenotype $A$ is characterized by the predominance of large, buoyant LDL particles (major LDL subclass peak between $260 \AA$ and $280 \AA$ ); and subclass phenotype $\mathrm{B}$ is characterized by predominance of small, dense LDL particles (major LDL subclass peak between $240 \AA$ and $255 \AA$ ). Subjects with subclass phenotype $B$ tend to have atherogenic lipoprotein profiles, including higher plasma triglycerides, very lowdensity lipoprotein (VLDL), and apoprotein B concentrations, and lower concentrations of apoprotein AI and high-density lipoprotein (HDL) (Austin 2000, Friedlander et al. 2000, Selby et al. 1993). Hypertriglyceridemia, low high-density lipoprotein cholesterol (HDL), and an increased fraction of small, dense LDL particles are frequent lipoprotein abnormalities noted in subjects with insulin resistance and type 2 diabetes mellitus (Selby et al. 1993, Austin et al. 1995, Syvanne and Taskinen 1997, Goff et al. 2005).

We are not aware of published reports concerning the risk of GDM in relation to maternal plasma LDL particle size. We therefore conducted the present study to address this gap in knowledge. Our a priori hypothesis was that the risk of GDM is increased with smaller maternal plasma LDL particle size.

\section{Methods}

Subjects for this analysis were recruited between April 1998 and June 2002 as part of a case-control study primarily designed to study the epidemiology of preeclampsia and gestational diabetes mellitus. The design of the study has been previously described (Sorensen et al. 2003). During the enrollment period, we identified women with GDM meeting the then-current diagnostic criteria described by the National Diabetes Data Group (NDDG) (Expert Committee 1997). Women were classified as having GDM if two or more of the following plasma glucose concentrations obtained during the 100 gram 3-hour OGTT test were abnormal: fasting $\geq 105 \mathrm{mg} / \mathrm{dl}$; 1 -hour $\geq 190 \mathrm{mg} / \mathrm{dl}$; 2-hour $\geq 165 \mathrm{mg} / \mathrm{dl}$; 3-hour $\geq 145 \mathrm{mg} / \mathrm{dl}$. Of the 288 eligible GDM cases identified, $233(81 \%)$ elected to provide written informed consent and to participate in this study. From the 233 women meeting the NDDG GDM definition, we randomly selected 110 subjects for the present study.

For the overall study, controls were women who remained normotensive and who did not meet the NDDG criteria for GDM. Among 750 eligible controls invited to participate, $50 \%$ agreed to provide written informed consent. Reasons for non-participation included not having time for the interview, having no interest in the goals of the study, and missed appointments. From the 375 available controls, we randomly selected 100 subjects to serve as controls in the current analysis. All selected cases and controls were normotensive and non-diabetic prior to the index pregnancy. Power and sample size calculations indicated that we had approximately $80 \%$ power to detect a 2.5 -fold increased risk of GDM ( $p=0.05$, two-tailed) associated with carriage of LDL subclass phenotype B using an estimated $5 \%$ prevalence of GDM and average $20 \%$. frequency of this phenotype. Previously reported frequencies range from 15 to $34 \%$ in non-Hispanic Whites (Selby et al. 1993).

During participants' postpartum hospital stay, trained interviewers administered a structured interview questionnaire to collect information on maternal sociodemographic, medical, reproductive and lifestyle characteristics. Participants were also asked to report their attained height and their weight three months prior to the index pregnancy. Pre-pregnancy body mass index (BMI), used as a measure of overall maternal adiposity, was calculated as weight (in kilograms) divided by height (in meters) squared. Maternal and infant medical records 
were reviewed to collect detailed obstetrical and prenatal information.

Maternal non-fasting blood samples, collected in EDTA $10 \mathrm{ml}$ Vacutainer tubes during the intrapartum period, were protected from ultraviolet light, kept on wet ice and processed within $30 \mathrm{~min}$ of phlebotomy. Plasma decanted into cryovials was preserved with metaphosphoric acid/dithiothreitol solution and frozen at $-70{ }^{\circ} \mathrm{C}$ until analysis. Blood samples were available for 105 cases and 96 controls for current analyses. Blood samples were also assayed for LDL cholesterol, HDL cholesterol, plasma triglycerides, and total cholesterol employing assays standardized by the Lipid Standardization Program of the Centers for Disease Control and Prevention, Atlanta, GA. The intra- and inter-assay coefficients of variation for the assay used were all bellow $10 \%$. All assays were performed without knowledge of pregnancy outcome.

Gradient gel electrophoresis was used to determine LDL phenotypes and LDL particle size. The procedures used have been described elsewhere (Austin et al. 1988). Briefly, 2-14\% polyacrylamide gels were produced, and electrophoresis procedures were applied by using plasma samples. A set of high-molecular-weight

Table 1. Distribution of gestational diabetes mellitus (GDM) cases and controls according to maternal selected characteristics, Seattle and Tacoma, Washington, USA, 1998-2002.

\begin{tabular}{|c|c|c|c|c|c|}
\hline \multirow[t]{2}{*}{ Characteristics } & \multicolumn{2}{|c|}{ GDM cases $(n=105)$} & \multicolumn{2}{|c|}{ Control subjects (n=96) } & \multirow[t]{2}{*}{$P$ value } \\
\hline & $\mathbf{n}$ & $\%$ & $\mathbf{n}$ & $\%$ & \\
\hline \multicolumn{6}{|l|}{ Maternal age (years) } \\
\hline$<35$ & 57 & 54.3 & 66 & 68.7 & 0.11 \\
\hline$\geq 35$ & 42 & 40.0 & 26 & 27.1 & \\
\hline Missing & 6 & 5.7 & 4 & 4.2 & \\
\hline Maternal Age (years) * & \multicolumn{2}{|c|}{$33.1 \pm 0.6$} & \multicolumn{2}{|c|}{$31.7 \pm 0.5$} & 0.08 \\
\hline \multicolumn{6}{|l|}{ Maternal race/ethnicity } \\
\hline Non-Hispanic White & 66 & 62.8 & 67 & 69.8 & 0.77 \\
\hline African American & 9 & 8.6 & 6 & 6.3 & \\
\hline Other & 23 & 21.9 & 18 & 18.8 & \\
\hline Missing & 7 & 6.7 & 5 & 2.1 & \\
\hline Not married & 15 & 14.3 & 12 & 12.5 & 0.81 \\
\hline$<12$ year education & 21 & 20.0 & 7 & 7.3 & 0.03 \\
\hline Nulliparous & 43 & 41.0 & 48 & 50.0 & 0.42 \\
\hline Smoked during pregnancy & 23 & 21.9 & 18 & 18.8 & 0.75 \\
\hline Family history of diabetes & 28 & 26.7 & 13 & 13.5 & 0.05 \\
\hline Physically inactivity during pregnancy & 50 & 47.6 & 34 & 35.4 & 0.15 \\
\hline \multicolumn{6}{|l|}{ Pre-pregnancy BMI $\left(\mathrm{kg} / \mathrm{m}^{2}\right)$} \\
\hline$<25$ & 31 & 29.5 & 70 & 72.9 & $<0.001$ \\
\hline$\geq 25$ & 67 & 63.8 & 21 & 21.9 & \\
\hline Missing & 7 & 6.7 & 5 & 5.2 & \\
\hline Pre-pregnancy BMI $\left(\mathrm{kg} / \mathrm{m}^{2}\right) *$ & \multicolumn{2}{|c|}{$29.5 \pm 0.7$} & \multicolumn{2}{|c|}{$22.8 \pm 0.4$} & $<0.001$ \\
\hline Time since last meal (hours) & \multicolumn{2}{|c|}{$2.2 \pm 0.3$} & \multicolumn{2}{|c|}{$2.4 \pm 0.4$} & 0.75 \\
\hline Gestational age at delivery (weeks) * & \multicolumn{2}{|c|}{$37.7 \pm 0.3$} & \multicolumn{2}{|c|}{$39.2 \pm 0.1$} & $<0.01$ \\
\hline
\end{tabular}

BMI: body mass index $=$ weight $/\left(\right.$ height $\left.^{2}\right), *$ Mean \pm standard error. 
Table 2. Maternal plasma lipid and lipoprotein according to gestational diabetes mellitus (GDM) case-control status in pregnant women, Seattle, Washington, USA 1998-2002.

\begin{tabular}{lccc}
\hline Measurement (mg/d) & GDM cases & Control subjects & P value* \\
$(\mathrm{n}=96)$ & & \\
\hline Total cholesterol & $182.3 \pm 4.2$ & $192.4 \pm 4.1$ & 0.09 \\
Low-density lipoprotein cholesterol & $87.8 \pm 3.3$ & $99.3 \pm 3.3$ & 0.01 \\
High-density lipoprotein cholesterol & $49.4 \pm 1.4$ & $56.3 \pm 1.3$ & $<0.001$ \\
Triglycerides & $223.2 \pm 8.7$ & $178.2 \pm 6.5$ & $<0.001$ \\
\hline
\end{tabular}

Data are means \pm S.E.M. $*$ P value from Student's $t$ test

Table 3. Odds ratios $(\mathrm{OR})$ and $95 \%$. confidence intervals $(\mathrm{CI})$ of gestational diabetes mellitus (GDM) according to low-density lipoprotein (LDL) phenotype and particle size, Seattle, Washington, USA 1998-2002.

\begin{tabular}{lcccc}
\hline LDL particle size & $\begin{array}{c}\text { GDM Cases } \\
(\mathrm{n}=105)\end{array}$ & $\begin{array}{c}\text { Control Subjects } \\
(\mathrm{n}=96)\end{array}$ & $\begin{array}{c}\text { Unadjusted } \\
\text { OR }(95 \% \mathrm{CI})\end{array}$ & $\begin{array}{c}\text { Adjusted } \\
\text { OR }(95 \% \mathrm{CI})\end{array}$ \\
\hline $\begin{array}{l}\text { LDL Phenotype } \\
\begin{array}{l}\text { Phenotype } A \\
\text { Phenotype B }\end{array}\end{array}$ & 95 & & & \\
& 10 & 2 & $4.0($ referent $)$ & 1.0 (referent) \\
$\begin{array}{l}\text { LDL particle size } \\
(\text { Mean } \pm S E)(\AA)\end{array}$ & $270.1 \pm 0.6$ & $272.7 \pm 0.6$ & $\begin{array}{c}2.1(1.3-3.4) \\
\text { for every } 10 \text { units } \\
\text { decrease }\end{array}$ & $\begin{array}{c}\text { for every } 10 \text { units } \\
\text { decrease }\end{array}$ \\
\hline
\end{tabular}

${ }^{1} \mathrm{OR}$ and $95 \%$. $\mathrm{Cl}$ adjusted for maternal age at pregnancy (categorical), nulliparity, family history of diabetes mellitus, gestational age at delivery and pre-pregnancy body mass index (categorical) as well as maternal plasma triglyceride concentrations.

standards was also run on each gel and was used to construct a quadratic calibration curve for estimating the diameter of LDL particle. In addition, two quality control samples with well-characterized LDL subclass phenotypes were run on each gel. Two gels were run on each sample by using whole plasma, and investigators were blinded to the case-control status of the study subject. The scan data for each sample lane on the gel were used to classify the LDL subclass phenotype on the basis of blinded evaluations by three reviewers and to estimate the diameter of the major LDL subclass (LDL particle size). More specifically, LDL subclass phenotype A is characterized by a predominance of large, buoyant LDL particles with a peak diameter of generally $>260 \AA$. In contrast, LDL subclass phenotype B is characterized by a predominance of small, dense LDL particles with a peak diameter of $\leq 255 \AA$. Phenotype I (Intermediate) is defined as major peak between $255 \AA$ and $260 \AA$. For statistical analyses requiring a dichotomous variable, subclass phenotype I subjects were grouped with subjects with subclass phenotype B. LDL particle size was defined as the estimated diameter of the major LDL subclass from the gels and was used as a continuous variable in the analyses.

Analyses were performed with LDL subclass phenotypes and LDL particle size as the exposures of interest. Student's $t$ tests for independent samples were used to compare mean lipid concentrations and LDL particle size between cases and controls. We employed logistic regression procedure (Rothman and Greenland 1998) to estimate the odds ratios (ORs) and $95 \%$ confidence intervals (CIs) of the relative risk of GDM associated with both LDL subclass phenotype and mean LDL particle size measured in $10-\AA$ increment. Multivariate analyses were initially performed with adjustment for maternal age at pregnancy, nulliparity, family history of diabetes mellitus, pre-pregnancy BMI and gestational age at delivery as well as maternal plasma triglyceride concentrations, specially. In the case of hypertriglyceridemia, facilitated transfer of triglycerides into LDL might occur and the subsequent hydrolysis of triglycerides by hepatic lipase may give rise to smaller 


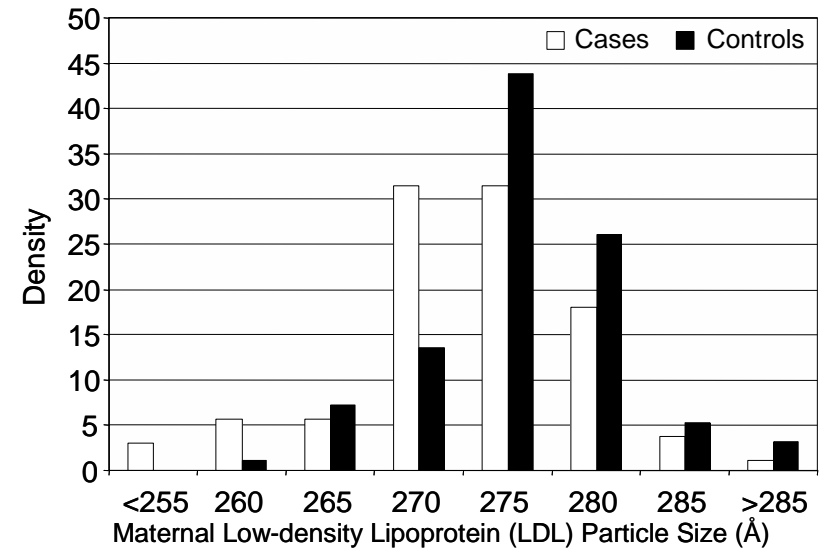

Fig. 1. Frequency distribution of low-density lipoprotein (LDL) particle size among gestational diabetes mellitus (GDM) cases and controls, Seattle, Washington, USA 1998-2002. (Mean values (standard error) for cases and controls were $270.1 \pm 0.6$ and $272.7 \pm 0.6 \AA$, respectively, $p=0.003$ ).

and denser LDL particles (Deckelbaum et al. 1984).

The procedures used in this study were in agreement with the protocols approved by the Institutional Review Boards of Swedish Medical Center and Tacoma General Hospital, respectively. All participants provided written informed consent.

\section{Results}

Compared with controls, GDM cases tended to be older and heavier (Table 1). Cases and controls did not differ with regard to smoking status and race/ethnicity. A higher proportion of cases than controls completed fewer than 12 years of education. Compared with controls, cases were also more likely to have a family history of diabetes. Table 2 shows the unadjusted mean lipid concentrations for cases and controls. Cases had significantly higher plasma triglyceride and lower HDL cholesterol concentration (all $\mathrm{p}<0.001$ ) compared with controls.

Although the proportion of women with the small, dense LDL (subclass phenotype B) was relatively low in this population $(2.1 \%$ in controls and $10.5 \%$ in cases); the proportion of cases with subclass phenotype $\mathrm{B}$ was significantly greater than the proportion of controls $(\mathrm{p}=0.04)$. Subclass phenotype B was associated with a significant 4.9-fold (95\% CI: 1.1-23.2) increased risk of GDM in univariate analyses. The odds ratio was attenuated somewhat, and became statistically insignificant, when we adjusted for maternal age, nulliparity, family history of diabetes, pre-pregnancy BMI, gestational age at delivery, and triglyceride concentration (adjusted $\mathrm{OR}=4.2,95 \%$ CI: $0.5-39.5$ ) (Table 3).

Frequency distributions of LDL particle size for cases and controls are presented in Figure 1. Mean LDL particle size was significantly lower in cases than in controls $(270.1 \pm 0.6$ vs. $272.7 \pm 0.6 \AA$, respectively, $\mathrm{p}=0.003$ ). Every $10-\AA \AA$ reduction in LDL particle size was associated with a 2-fold increased risk of GDM (OR=2.1, $95 \%$ CI: 1.3-3.4). The association was slightly attenuated and became statistically insignificant after adjusting for other potential confounders including plasma triglyceride concentrations ( $\mathrm{OR}=1.8,95 \%$ CI: 0.9-3.3).

\section{Discussion}

In this case-control study, we showed that LDL subclass phenotype B (predominance of small, dense LDL particles) was associated with a 4-fold increased risk of GDM. To our knowledge there are no published studies concerning the relation between maternal LDL particle size and GDM risk. Our findings are generally consistent with a relatively large body of literature (mostly case-control studies) documenting the association of small, dense LDL particle with insulin resistance (Friedlander et al. 2000, Selby et al. 1993, Goff et al. 2005) and type 2 diabetes (Syvanne and Taskinen 1997, Austin et al. 1995, Siegel et al. 1996, Gray et al. 1997) in non-pregnant individuals. Goff et al. (2005) analyzed data from the Insulin Resistance Atherosclerosis Study and noted that insulin sensitivity was positively associated with LDL size $(\rho=0.34, \mathrm{P}<0.001)$. In addition, these authors noted that insulin sensitivity was associated with triglycerides $(\rho=-0.36, \mathrm{P}<0.001)$ and HDL $(\rho=0.37$, $\mathrm{P}<0.001)$. Siegel et al. (1996) demonstrated that diabetic patients in the Framingham offspring study had lower HDL, higher triglycerides, and higher LDL particle scores (indicating smaller size) than non-diabetics in both sexes. The presence of LDL subclass phenotype B was highly associated with diabetes and hypertriglyceridemia in both sexes, and the OR remained significant in women $(\mathrm{OR}=2.6, \mathrm{p}=0.009)$ but not in men $(\mathrm{OR}=1.4, \mathrm{p}=0.13)$ after adjustment for age and hypertriglyceridemia. Austin et al. (1995) analyzed a prospective nested case-control sample of 204 elderly Finland men and women and noted that subjects with a predominance of small, dense LDL had a greater than twofold increased risk for developing type $2 \mathrm{DM}$ over the 3.5-year follow-up period. This association was independent of age, sex, glucose intolerance, and BMI but was not independent of fasting 
triglyceride or insulin levels. Furthermore, an increase of $5 \AA$ in LDL diameter was associated with a $16 \%$ decrease in risk of NIDDM; and a composite variable of LDL diameter and triglyceride and HDL cholesterol concentrations was also associated with NIDDM. These associations may be attributable to the role of LDL subclass phenotype $\mathrm{B}$ as a marker for insulin resistance.

As we mentioned, the proportion of women with small, dense LDL was low in this study population ( $2.1 \%$ in controls and $10.5 \%$ in cases, respectively). In one study including 10 women with uncomplicated primiparous pregnancy, non-smoker and no history of metabolic disorders, Hubel et al. (1998) showed that LDL diameter decreased progressively with advancing gestation, evident by 16-20 weeks relative to 5-12 weeks. The average diameter decrease from early to late gestation was $13 \AA$. Across gestational age, LDL diameter correlated inversely with triglycerides $(\rho=-0.61$, $\mathrm{P}<0.001$ ). By comparison to the women in Hubel's study, women in the current study were less dyslipidemic at late gestation [e.g. total cholesterol: $192.4 \mathrm{mg} / \mathrm{dl}$ (current study) vs. $296 \mathrm{mg} / \mathrm{dl}$; triglycerides: $178.2 \mathrm{mg} / \mathrm{dl}$ (current study) vs. $272 \mathrm{mg} / \mathrm{dl}]$. Underlying demographic and population differences between the two studies may have resulted in difference in the LDL phenotype between the two studied populations.

Several limitations must be considered when interpreting the results of our study. First, given that we collected blood samples after GDM was diagnosed, we cannot with certainty determine whether the observed differences in plasma LDL particle size preceded the onset of GDM, or whether the differences may be attributed to physiological alterations such as increased lipid peroxidation or oxidative stress secondary to the disorder. Prospective studies with serial measurements of plasma LDL particle size and glycemic control throughout pregnancy are required to confirm and expand upon our observations. Second, we cannot exclude the possibility of selection bias. The control participation rate was $50 \%$ and the case participation rate was $81 \%$. However, the characteristics of participating controls appeared to be similar to those of all women delivering at the study hospitals by state birth certificate data. Moreover, it is unlikely that participation was conditional on LDL subclass phenotype. Third, variation in lipid measurements may have been introduced by aspects of the study design. Specifically, women were not asked to fast before blood collection. However, women were questioned regarding elapsed time since eating at the blood collection and adjustment for this variable did not change results of our analyses. Fourth, the diagnostic criteria for GDM used for our study were changed in 2002, so that lower plasma glucose concentrations obtained during the OGTT test would now be considered as the diagnosis cut-point (fasting $\geq 95 \mathrm{mg} / \mathrm{dl}$; 1-hour $\geq 180 \mathrm{mg} / \mathrm{dl}$; 2-hour $\geq 155 \mathrm{mg} / \mathrm{dl}$; 3-hour $\geq 140 \mathrm{mg} / \mathrm{dl}$ ) (Expert Committee 2002). If the new, more inclusive criteria were used in our study, some women, who were classified as controls in these analyses, would have been classified as having GDM, which might lead to a weakened association. Lastly, maternal pregestational diabetes status was based on self-report and confirmed in medical records, those women were excluded from the analysis. Our study subjects are comprised of welleducated, upper income women with $>95 \%$ reporting to have had regular medical examinations within 24 months of pregnancy. Thus it is unlikely that a large proportion of these women had undiagnosed type 2 diabetics.

The association between GDM risk and LDL subclass phenotype B is biologically plausible. Haffner (1998) and Ferrannini et al. (1992) provided evidence that the contributions of compensatory hyperinsulinemia and insulin resistance are major causes of dyslipidemia in diabetic patients. Biological mechanisms for the observed associations are currently unknown. A possible explanation is that the small, dense LDL is genetically determined and results directly from the remodeling of certain VLDL subfractions (Esteve et al. 2004). Another possibility might involve facilitated transfer of triglycerides into LDL in the case of hypertriglyceridemia in diabetic patients (Deckelbaum et al. 1984); the subsequent hydrolysis of triglycerides by hepatic lipase may give rise to smaller and denser LDL particles. The modification of LDL particle may also involve increased oxidative stress (Hidaka et al. 2005). Alternatively, small dense LDL particles have atherosclerotic potential that may exacerbate the risk of developing GDM. For instance, investigators have reported that small, dense LDL particles increase affinity for the LDL receptor (Galeano et al. 1998), susceptibility to oxidation (Tribble et al. 2001), vascular permeability and the concentrations of fat-laden cells in the vascular media (Aviram and Maor 1992).

In summary, our results suggest that small, dense LDL particle is associated with a 4.9-fold increased risk of GDM (95 \% CI: 1.1-23.2). The association was only slightly attenuated when adjustments were made for triglyceride, a correlate of LDL particle size, and other 
confounders $(\mathrm{OR}=4.2,95 \% \mathrm{CI}: 0.5-39.5)$, although this result did not achieve statistical significance. These multivariate findings suggest that LDL subclass phenotype B may be associated with GDM risk. However, larger studies and prospective studies that include collection of pre-diagnostic blood samples are needed to more precisely measure the independent associations of LDL size, hypertriglyceridemia as well as other clustered risk factors of insulin resistance and impaired insulin secretion in pregnant women.

\section{Acknowledgements}

This research was supported in part by awards from the National Institutes of Health (HD/HL R01 34888 and HD/HL R01 32562. The authors are indebted to the participants of the ALPHA Study for their cooperation. They are also grateful for the technical expertise contributed by staff in Center for Perinatal Studies and Ann Wilson, Carrie Nelson and Kate Chilson from Dr. Austin's Laboratory at the University of Washington, School of Public Health and Community Medicine.

\section{References}

ABOUGHALIA AH, KHATER LM, ADB El-WAHED MA, EL-BADRAWY MF: Lipoprotein (a) and lipid profile in neonates from mothers with three different types of diabetes mellitus. Clin Biochem 36: 563-569, 2003.

AMERICAN DIABETES ASSOCIATION: Gestational diabetes mellitus. Diabetes Care 22 (Suppl): 74-76, 1999.

AUSTIN MA: Triglyceride, small, dense low-density lipoprotein, and the atherogenic lipoprotein phenotype. Curr Atheroscler Rep 2: 200-207, 2000.

AUSTIN MA, BRESLOW JL, HENNEKENS CH, BURING JE, WILLETT WC, KRAUSS RM: Low-density lipoprotein subclass patterns and risk of myocardial infarction. JAMA 260: 1917-1921, 1988.

AUSTIN MA, MYKKANEN L, KUUSISTO J, EDWARDS KL, NELSON C, HAFFNER SM, PYORALA K, LAAKSO M: Prospective study of small LDLs as a risk factor for non-insulin dependent diabetes mellitus in elderly men and women. Circulation 92: 1770-1778, 1995.

AVIRAM M, MAOR I: Phospholipase A2-modified LDL is taken up at enhanced rate by macrophages. Biochem Biophys Res Commun 185: 465-472, 1992.

BEN-HAROUSH A, YOGEV Y, HOD M: Epidemiology of gestational diabetes mellitus and its association with Type 2 diabetes. Diabet Med 21: 103-113, 2004.

COUSTAN DR, CARPENTER MW, O'SULLIVAN PS, CARR SR: Gestational diabetes: predictors of subsequent disordered glucose metabolism. Am J Obstet Gynecol 168: 1139-1145, 1993.

DAMM P: Gestational diabetes mellitus and subsequent development of overt diabetes mellitus. Danish Med Bulletin 45: 495-509, 1998.

DECKELBAUM RJ, GRANOT E, OSCHRY Y, ROSE L, EISENBERG S. Plasma triglyceride determines structurecomposition in low and high density lipoproteins. Arteriosclerosis 4: 225-231, 1984.

ENQUOBAHRIE DA, MILLIAMS MA, QIU C, LUTHY DA: Early pregnancy lipid concentrations and the risk of gestational diabetes mellitus. Diabetes Res Clin Pract 70: 134-142, 2005.

ESTEVE E, FAURE E, CALVO F, AGUILlO E, BLASCO C, ROCHE MJ, MOZAS P, POCOVI M: SNP3 polymorphism in apo A-V gene is associated with small dense LDL particles in Type 2 diabetes. Diabetologia 47: 355-356, 2004.

EXPERT COMMITTEE ON THE DIAGNOSIS AND CLASSIFICATION OF DIABETES MELLITUS: Report. Diabetes Care 20: 1183-1197, 1997.

EXPERT COMMITTEE ON THE DIAGNOSIS AND CLASSIFICATION OF DIABETES MELLITUS: Report. Diabetes Care 25 (Suppl): 5-20, 2002.

FERRANNINI E, STERN MP, GALVAN AQ, MITCHELL BD, HAFFNER SM: Impact of associated conditions on glycemic control of NIDDM patients. Diabetes Care 15: 508-514, 1992.

FRIEDLANDER Y, KIDRON M, CASLAKE M, LAMB T, MCCONNELL M, BAR-ON H: Low density lipoprotein particle size and risk factors of insulin resistance syndrome. Atherosclerosis 148: 141-149, 2000.

GALEANO FN, AL-HAIDERI M, KEYSERMAN F, RUMSEY SC, DECKELBAUM RJ: Small dense low density lipoprotein has increased affinity for LDL receptor-independent cell surface binding site: a potential mechanism for increased atherogenicity. J Lipid Res 39: 1263-1273, 1998. 
GOFF DC, JR, D'AGOSTINO RB JR, HAFFNER SM, OTVOS JD: Insulin resistance and adiposity influence lipoprotein size and subclass concentrations. Results from the Insulin Resistance Atherosclerosis Study. Metabolism 54: 264-270, 2005.

GRAY RS, ROBBINS DC, WANG W, YEH JL, FABSITZ RR, COWAN LD, WELTY TK, LEE ET, KRAUSS RM, HOWARD BV: Relation of LDL size to the insulin resistance syndrome and coronary heart disease in American Indians. The Strong Heart Study. Arterioscler Thromb Vasc Biol 17: 2713-2720, 1997.

HAFFNER SM: Management of dyslipidemia in adults with diabetes. Diabetes Care 21: 160-178, 1998.

HIDAKA A, INOUE K, KUTSUAKE S, ADACHI M, KAKUTA Y, KOJO S: Decrease in the particle size of lowdensity lipoprotein (LDL) by oxidation. Bioorg Med Chem Lett 15: 2781-2785, 2005.

HUBEL CA, SHAKIR Y, GALLAHER MJ, MCLAUGHLIN MK, ROBERTS JM: Low-density lipoprotein particle size decreases during normal pregnancy in association with triglyceride increases. J Soc Gynecol Investig 5 : 244-250, 1998.

KRAUSS RM, BURKE DJ: Identification of multiple subclasses of plasma low density lipoproteins in normal humans. J Lipid Res 23: 97-104, 1982.

LAUENBORG J, MATHIESEN E, HANSEN T, GLUMER C, JORGENSEN T, BORCH-JOHNSEN K, HORNNES P, PEDERSEN O, DAMM P: The prevalence of the metabolic syndrome in a Danish population of women with previous gestational diabetes mellitus is threefold higher than in the general population. J Clin Endocrinol Metab 90: 4004-4010, 2005.

O'SULLIVAN JB: Long term follow-up of gestational diabetes. In: Early Diabetes. CAMERINI DAVALOS RA, COLE HS (eds), Academic Press, New York, 1984, pp 1009-1027.

ROTHMAN KJ, GREENLAND S: Modern Epidemiology, Lippincott-Raven Publishers, Philadelphia, 1998.

SELBY JV, AUSTIN MA, NEWMAN B, ZHANG D, QUESENBERRY CP JR, MAYER EJ, KRAUSS RM: LDL subclass phenotypes and the insulin resistance syndrome in women. Circulation 88: 381-387, 1993.

SIEGEL RD, CUPPLES A, SCHAEFER EJ, WILSON PW: Lipoproteins, apolipoproteins, and low-density lipoprotein size among diabetics in the Framingham offspring study. Metabolism 45: 1267-1272, 1996.

SORENSEN TK, WILLIAMS MA, LEE IM, DASHOW EE, THOMPSON ML, LUTHY DA: Recreational physical activity during pregnancy and risk of preeclampsia. Hypertension 41: 1273-80, 2003.

SYVANNE M, TASKINEN MR: Lipids and lipoproteins as coronary risk factors in non-insulin-dependent diabetes mellitus. Lancet 350 (Suppl 1): 20-23, 1997.

TRIBBLE DJ, RIZZO M, CHAIR A, LEWIS DM, BLANCHE PJ, KLAUSS RM: Enhanced oxidative susceptibility and reduced antioxidant content of metabolic precursors of small, dense low-density lipoproteins. Am J Med 110: $103-110,2001$.

\section{Corresponding author}

Chunfang Qiu, Swedish Medical Center (Center for Perinatal Studies), 1124 Columbia Street, Suite 750, Seattle, WA 98104. Fax: 206-215-6995. E-mail: Chun-fang.Qiu@Swedish.org 